\title{
Cognitive concomitants of dopamine system stimulation in Parkinsonian patients
}

\author{
ERICH MOHR, * GIOVANNI FABBRINI, $\dagger$ STEFANO RUGGIERI, PAUL FEDIO,* \\ THOMAS N CHASE $\dagger$
}

From the Medical Neurology Branch, ${ }^{*}$ Experimental Therapeutics Branch, $\dagger$ National Institute of Neurological and Communicative Disorders and Stroke, Bethesda, MD, USA, and the Prima Clinica Neurologica Universita, degli Studi La Sapienza, $\ddagger$ Rome, Italy

SUMMARY Verbal, visuospatial and motor functions were studied in eight Parkinsonian patients both during levodopa stimulated and unstimulated state and in eight matched, untreated, healthy controls. Profound changes in patients' motor status were accompanied by relatively selective effects on delayed verbal memory, a function which was also most impaired compared with controls. With dopaminomimetic therapy, tests of delayed verbal memory consistently improved, but did not reach control performance levels. These results could indicate a functional impairment in the mesocortical dopamine system, which can be attenuated, but not entirely corrected, by dopaminomimetic therapy.

Considerable evidence suggests that dopamine containing neurons participate in the regulation of certain cognitive processes. In the experimental animal, procedures which attenuate central dopaminergic function have been reported to compromise delayed spatial alternation tasks, ${ }^{1}$ acquisition of active avoidance $^{2}$ and maintenance of normal spatial working memory. ${ }^{3}$ Pharmacological stimulation of the dopamine system, on the other hand, tends to reverse some of these performance deficits. ${ }^{4-6}$ In man, dopaminergic hypofunction has been implicated in the pathogenesis of Alzheimer's dementia, ${ }^{7}$ as well as in certain intellectual deficits occurring in Parkinson's disease. ${ }^{8}$ Clinical studies suggest that cognitive deficits in Parkinsonian patients involve mainly memory and learning. ${ }^{9}$ Specifically, while semantic or knowledge memory, notably context-free general principles, associations and rules ${ }^{10}$ is reportedly unimpaired, performance in the realm of episodic memory, notably context-bound knowledge, including information learned in the laboratory, ${ }^{10}$ may be compromised in untreated Parkinsonian individuals. ${ }^{11}$ Conversely, although reports of cognitive

Address for reprint requests: Dr Erich Mohr, National Institutes of Health, 9000 Rockville Pike, Building 10, Room 5C 416, Bethesda, MD 20892, USA.

Received 30 April 1986 and in revised form 31 December 1986. Accepted 5 January 1987 improvement with levodopa treatment in Parkinsonian patients are equivocal, ${ }^{12-14}$ treatment with dopaminomimetics has been reported to improve effortful memory in normal individuals. ${ }^{15}$

Notwithstanding these observations, the precise contribution of the dopamine system to defined aspects of human intellectual function remains uncertain. In an attempt to explore this matter, verbal and visuospatial functions were studied in Parkinsonian patients both during levodopa stimulated and unstimulated states.

\section{Methods}

Eight patients (five men, three women; mean \pm SEM age $58 \pm 4.7$ years, range $29-70$ ), with the diagnosis of idiopathic Parkinson's disease and eight normal control subjects (five men, three women, mean \pm SEM age $59 \pm 3 \cdot 1$ years, range 30-71), consented to participate in this study after full disclosure of its purpose, risks and potential benefits. Symptoms had been present in the patient group for 2 to 21 years $(11 \cdot 1 \pm 2 \cdot 1)$ and unmedicated severity ranged from 2 to 4 on the Hoehn and Yahr Scale. ${ }^{16}$ All patients had received levodopa for 1 to 12 years (mean 7.9 \pm 1.6 ). Each now manifested consistent wearing-off phenomena, characterised by a loss of motor response 2 to 4 hours after levodopa administration. All normal control subjects underwent a routine neurological and neuropsychological evaluation to insure that no significant illness was present.

All study subjects received an extensive assessment of intellectual and memory functions. Psychometric tests of 
intelligence during optimal levodopa treatment, revealed low to high average functioning in the patient group, whose educational level ranged from 12 to 20 years $(15 \pm 1 \cdot 0)$ : Wechsler Adult Intelligence Scale-Revised ${ }^{17}$ Full Scale IQ $100 \pm 3.0$ (range 92-115); Verbal IQ $104 \pm 4.1$ (range 88-120); Performance IQ $97 \pm 3.7$ (range 74-107); Wechsler Memory Scale ${ }^{18} 111 \pm 5 \cdot 6$ (range 95-141); Mattis Dementia Rating Scale ${ }^{19} 137 \pm 2 \cdot 2$ (range 126-143). The normal control group (mean education $13 \cdot 5 \pm 0 \cdot 4$, range $12-16$ years), by comparison showed average to superior intellectual and mnemonic function (Full Scale IQ $115.5 \pm 2 \cdot 8$, range 103-141; Verbal IQ $114 \pm 2 \cdot 6$, range 98-134; Performance IQ $114.6 \pm 3.3$, range 95-135; Memory Quotient $121 \cdot 6 \pm 3 \cdot 6$, range 101-143). The Mattis dementia rating scale revealed no evidence of dementia in the controls (141 $\pm 0 \cdot 4$, range $139-144)$.

Throughout the study, patients received levodopacarbidopa (Sinemet) each at their optimal antiparkinsonian dose ( 125 to $250 \mathrm{mg}$ per dose of levodopa) every 2 to 4 hours while awake. Overall motor function was monitored by the patient and by a neurologist, familiar with the subject's individual response to antiparkinsonian treatment; both were asked to identify on periods during which Parkinsonian signs abated and dyskinesias sometimes appeared, from off periods, when Parkinsonian signs returned.

Motor and cognitive function were then assessed by a neurologist and a neuropsychologist who had no prior knowledge of these designations. Parkinsonian and dyskinesia severity was evaluated first, each on a scale of 0 (absent) to 4 (very severe); venous blood was then drawn for the determination of plasma levodopa levels; finally neuropsychologic testing was carried out. Data were analysed only from those sessions during which motor function remained stable. The $\mathbf{5 0}$ minute assessment sessions during on and off periods occurred in random order, over 4 nonconsecutive days.

Neuropsychological tests were selected specifically to sample those domains reportedly impaired in untreated Parkinsonian patients, in order to optimise chances of detecting treatment related changes in cognition. These included in particular memory, learning and visuospatial orientation. ${ }^{112426}$ Alternative forms of the following neuro- behavioural instruments were administered in such a manner that speed or accuracy of motor performance did not affect scoring:

Verbal Fluency Patients were given 60 seconds to name as many supermarket items as possible. ${ }^{19}$

Embedded Figures Test Patients were asked to recognise a simple geometric design in one of four complex patterns. ${ }^{20}$ Logical Memory The narrative account of a complex event had to be recalled verbatim. ${ }^{18}$

Paired Associate Words A four trial series (three immediate, one 30 minute delayed recall) of six easy paired associates (for example, gold-silver) and four hard paired associates (such as school-grocery) were read to patients in 1 second intervals. After each series, patients were furnished with the first word of the pair and asked to recall the correct associate. ${ }^{18}$

Visual Form Discrimination Test A set of figures was presented for 10 seconds and patients were asked to select the correct set among four alternative designs. ${ }^{21}$ In the delayed recall portion of this test, a 15 second interval separated the initial presentation and the multiple choice condition.

All data are presented as the mean \pm SEM and were statistically evaluated by standard repeated measures Analysis of Variance procedures ${ }^{22}$ and when appropriate, with correlational measures. ${ }^{23}$

\section{Results}

Motor function evidenced a consistent $(p<0.001)$ response to levodopa therapy (table). During on periods, as identified by patient and physician global assessments, plasma drug levels averaged nearly 3.5 fold higher $(5.5 \pm 1.2 \mu \mathrm{g} / \mathrm{ml})$ than during off periods $(1.6 \pm 0.71 \mu \mathrm{g} / \mathrm{ml} ; \mathrm{p}<0.001)$. Patients' and physician's assessment of on and off state were in perfect agreement. While in the on state, neurologists' ratings indicated that Parkinsonian signs were absent to mild with scores ranging from 0 to 1 , while dyskinesias were absent to moderately severe, with scores between

Table Effect of levodopa on motor and cognitive function

\begin{tabular}{|c|c|c|c|c|}
\hline & \multirow[b]{2}{*}{ Maximum score } & \multicolumn{2}{|c|}{ Parkinsonian patients } & \multirow[b]{2}{*}{ Controls } \\
\hline & & off & On & \\
\hline \multicolumn{5}{|l|}{ Motor effects: } \\
\hline Parkinsonian score & 4 & $1.8 \pm 0.15$ & $0.55 \pm 0.06^{*}$ & - \\
\hline Dyskinesia score & 4 & $0 \pm 0$ & $1.3 \pm 0.38^{*}$ & - \\
\hline \multicolumn{5}{|c|}{ Learning-dissociated effects: } \\
\hline Verbal fluency & - & $16 \pm 1 \cdot 1$ & $\pm 1 \cdot 1$ & $19 \cdot 2 \pm 1 \cdot 7$ \\
\hline \multicolumn{5}{|c|}{ Immediate memory effects: } \\
\hline Logical memory & 23 & $4.4 \pm 0.68$ & $4.9 \pm 0.77$ & $9.0 \pm 0.67 \dagger$ \\
\hline Paired associates & 7 & $3.4 \pm 0.22$ & $3.9 \pm 0.31$ & $5.1 \pm 0.26 t$ \\
\hline Visual retention & 4 & $2.6 \pm 0.37$ & $3.1 \pm 0.27$ & $3.2 \pm 0.21$ \\
\hline \multicolumn{5}{|l|}{ Delayed memory effects: } \\
\hline Logical memory & 23 & $2.9 \pm 0.53$ & $4.0 \pm 0.46 \pm$ & $7.3 \pm 0.61 \dagger$ \\
\hline Paired associates & 7 & $3.8 \pm 0.37$ & $4.6 \pm 0.55 \ddagger$ & $5.7 \pm 0.21 \dagger$ \\
\hline Visual retention & 4 & $2.7 \pm 0.24$ & $2.8 \pm 0.31$ & $3.1 \pm 0.15$ \\
\hline
\end{tabular}

* $p<0.001 ; \pm p<.05$ for difference from off period by ANOVA for repeated measures.

$t \mathrm{p}<0.01$ for difference between controls and Parkinsonian patients both during "on" and "off" state. 
between 0 and 3 (table). In contrast, during off periods, Parkinsonian scores ranged from mild to moderately severe (1 to 3 ), while no patient manifested dyskinesia.

Cognitive function exhibited a more complex pattern of response to levodopa stimulation (table). Neuropsychological tests which are largely dissociated from the context of learning (Verbal Fluency and Embedded Figures), yielded no differences between on and off periods. Similarly, tasks involving immediate memory, both in the verbal and the visuospatial realm, also failed to discriminate between the two states.

Delayed memory tests did however suggest differences between the on and off periods (table). Average performance on the Logical Memory and the Paired Associates tasks showed a small, albeit consistent, improvement during the levodopa stimulated state $(p<0.05)$. For the individual patient, six of eight had a performance increment on the Logical Memory task, while five of eight improved on the Paired Associates test (fig 1). Analysis of the Paired Associates results revealed essentially identical responses between the on and off states on the first trial and the appearance of differences only on subsequent trials (fig 2). There was no significant correlation between the motor response to levodopa and the cognitive response as measured by either of these tests. Significant alterations in delayed memory could be documented only on the verbal tasks. The Visual Retention test for visuospatial memory failed to demonstrate any consistent differences (table): four patients improved, one remained unchanged and three deteriorated.

A comparison of cognitive performance patterns in Parkinsonian patients with those in normal controls, revealed significant decrements in the patient group of all those functions showing significant differences between the on and off states $(p<0.01)$. This held true both for comparison of normals with patients when they were "on" as well as when they were "off" (table). Conversely, with the exception of visual disembedding (Embedded Figures), none of the tasks showing "no differences" between patients' on and off period, evidenced significant performance decrements compared to control scores.

\section{Discussion}

The present results support the view that pharmacological activation of the dopamine system can affect intellectual function in patients with Parkinson's disease. ${ }^{132425}$ Individuals with predictable wearing-off phenomena were chosen for study so that periods of levodopa-induced stimulation of central dopaminergic mechanisms could be reliably distinguished from
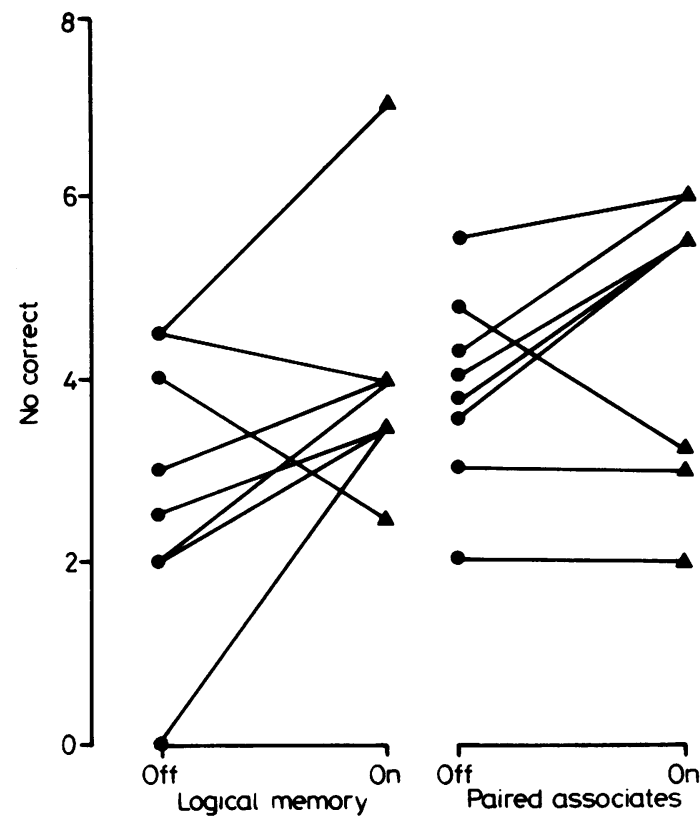

Fig 1 Delayed memory effects: Logical Memory and Paired Associates-individual scores.

periods in which motor function returned to its untreated state. The on-off states were studied in random order on non-consecutive days, in order to limit the possibility of result contamination due to extraneous factors. The assertion that neuropsychological tests used in this study were essentially unaffected by the patients' motor function, gained some empirical support from the absence of any cor-

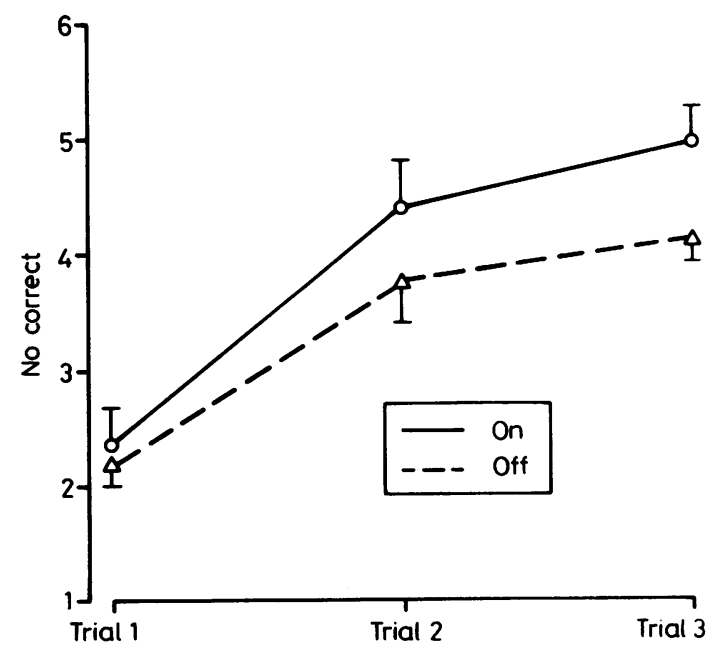

Fig 2 Paired Associate learning by trial. 
relation between the degree of levodopa-induced change in motor performance and the magnitude of change in the cognitive measures.

Our finding that levodopa stimulation exerts relatively selective effects on delayed memory function is in agreement with an earlier case study, ${ }^{26}$ where it was ascertained that the on-off phenomenon did not influence initial encoding as dramatically as retention of material over time. The present, relatively selective findings stand in contrast, however, with those of other studies, which emphasised a more general effect of levodopa on both affect and arousal, when comparing the on with the off state. ${ }^{2427}$ The fact that our patients appeared to manifest relatively selective cognitive changes, argues against their being attributable to a general alerting effect of levodopa. Indeed, significant levodopa-induced effects on cognitive function were observed in only two tests of delayed verbal memory, Logical Memory and Paired Associates. Both of these neurobehavioural tests evaluate episodic memory, a mnemonic function purportedly impaired in untreated, mildly to moderately affected, Parkinsonian patients. ${ }^{11}$ Specifically, it has been proposed that two different cognitive operations are involved in episodic memory; those that require sustained effort and cognitive capacity and those that are automatic and require only limited cognitive capacity. ${ }^{28}$ Weingartner et $a l^{11}$ ascertained that compromises in episodic memory function predominantly occurred in the realm of effortful processing. If one considers delayed recall conditions particularly effortful, it is not surprising that cognitive corollaries of the on-off phenomenon involve especially those functions. ${ }^{24}{ }^{26}$ This contention finds further support from the report of improved effortful memory in normal elderly individuals with levodopa stimulation. ${ }^{15}$ Finally, the fact that the single test of delayed visualspatial memory employed in this study, Visual Retention, revealed less consistent changes between the on and off states, might merely reflect the relatively small number of trials used in its administration.

Comparison of patient test scores with those of control subjects revealed that functions significantly improved by dopaminomimetic therapy were those most impaired in the Parkinsonian group. Since this deficit was evident both during on and off conditions, it would appear that cognitive decrements during the off state can be attenuated with dopaminomimetic therapy, but not restored to normal levels. Those neurobehavioural functions not evidencing consistent differences between on and off states, also showed no significant compromise compared to control scores, thus were evidently relatively unaffected by the disease. The sole exception, visual disembedding, might be deficient for reasons unrelated to hypofunction of the dopamine system.
Recent investigations in the experimental animal further support the concept that the dopamine system might be involved in memory functioning. The dopamine antagonist, haloperidol, reportedly disrupts both working and reference memory for a search task in rats with mesocortical damage. ${ }^{29}$ Lesioning of rat mesocortical and mesolimbic dopaminergic projections from the A-10 nucleus impaired retention of delayed alternation, ${ }^{1}$ while catecholamine depletion in the pre-frontal cortex of Rhesus monkeys reduced learning on a similar task, a deficit reversible with administration of levodopa. ${ }^{4}$ Moreover, biochemical results suggest that cortical dopamine containing neurons facilitate learning of a delayed alternation task in non-drug treated animals. ${ }^{30}$

Observations deriving from this study could have important implications for the treatment of specific cognitive deficits in both Parkinsonian and nonparkinsonian individuals. Clearly, the impact of antiparkinsonian therapy on extrapyramidal motor function is much more impressive than on cognitive performance, a finding which is in agreement with earlier assertions. ${ }^{2426}$ The profound changes in motor performance probably reflect hypofunction of the nigrostriatal dopaminergic system, while the relatively mild cognitive differences between on and off state might indicate a compromise in the functional integrity of another part of the dopaminergic system, particularly the mesocortical pathway. Indeed, the mesocortical system has been implicated in the pathophysiology of Parkinsonism both by direct biochemical evidence ${ }^{3132}$ as well as by the indirect neurobehavioural evidence presented here and elsewhere. ${ }^{2427}$ Taken together, the mesocortical system may well account for certain aspects of the cognitive decline in some Parkinsonian patients, particularly in the delayed recall of newly learned materials. It remains to be determined whether Parkinsonian patients more cognitively impaired than present subjects would derive greater benefit from dopaminomimetic treatment on these delayed memory tasks. Finally, if such therapeutic intervention can ameliorate certain aspects of memory failure in Parkinson's disease, the possibility of mesocortical dopamine system dysfunction in other disorders may merit further investigative attention.

\section{References}

1 Simon H, Scatton B, Le-Moal M. Dopaminergic A10 neurons are involved in cognitive functions. Nature 1980;286:150-1.

2 Koob GF, Simon H, Herman JP, Le Moal M. Neuroleptic-like disruption of the conditioned avoidance response requires destruction of both the mesolimbic and nigrostriatal dopamine systems. Brain Res 1984;303:319-29.

3 Beatty WW, Rush JR. Spatial working memory in rats: effects of 
monoaminergic antagonists. Pharmacol Biochem Behav 1983;18:7-12.

4 Brozoski TJ, Brown RM, Rosvold HE, Goldman PS. Cognitive deficits caused by regional depletion of dopamine in prefrontal cortex of rhesus monkey. Science 1979;205:929-31.

5 Seiden LS, Peterson DD. Reversal of the reserpine-induced suppression of the conditioned avoidance response by L-Dopa: Correlation of behavioral and biochemical differences in two strains of mice. $J$ Pharmacol Exp Ther 1968;159(2):422-8.

6 Zis AP, Fibiger HC, Phillips AG. Reversal by L-Dopa of impaired learning due to destruction of the dopaminergic nigro-striatal projection. Science 1974;185:960-2.

7 Gottfries CG. Neurotransmitters in the brains of patients with dementia disorders. Dan Med Bull 1985;32(1):44-8.

8 Agid Y, Ruberg M, Dubois B, Javoy-Agid F. Biochemical substrates of mental disturbances in Parkinson's disease. Adv Neurol 1984;40:211-8.

9 Mortimer JA, Pirozzolo FJ, Hansch EC, Webster DD. Relationship of motor symptoms to intellectual deficits in Parkinson's disease. Neurology 1982;32:133-7.

10 Squire L, Cohen NJ. Human memory and amnesia. In: Lynch G, McGaugh JL, Weinberger NM, eds. Neurobiology of learning and memory. New York: Guilford Press, 1984:3-64.

11 Weingartner H, Burns S, Diebel R, LeWitt PA. Cognitive impairments in Parkinson's disease: Distinguishing between effort-demanding and automatic cognitive processes. Psychiatry Res 1984;11:223-35.

12 Meier MJ, Martin WE. Intellectual changes associated with levodopa therapy. JAMA 1970;213(3):465-6.

13 Marsh GG, Markham CM, Ansel R. Levodopa's awakening effect on patients with Parkinsonism. J Neurol Neurosurg Psychiatry 1971;34:209-18.

14 Bowen FP, Kamienny RS, Burns MM, Yahr MD. Parkinsonism: Effects of levodopa treatment on concept formation. Neurology 1975;25:701-4.

15 Newman RP, Weingartner H, Smallberg SA, Calne DB. Effortful and automatic memory: Efforts of dopamine. Neurology 1984;34:805-7.

16 Hoehn MM, Yahr MD. Parkinsonism-onset, progression and mortality. Neurology 1967;17:427-42.

17 Wechsler D. Wechsler Adult Intelligence Scale-Revised. New York: The Psychological Corporation, 1981.

18 Wechsler D, Stone CP. Wechsler Memory Scale. New York: The Psychological Corporation, 1945.
19 Mattis S. Mental status examination for organic mental syndrome in the elderly. In: Bellack L, Karasu TB, eds. Geriatric Psychiatry. New York: Grune and Stratton, 1976:77-121.

20 Kapur N, Butters N. Visuoperceptive deficits in long-term alcoholics and alcoholics with Korsakoff's psychosis. J Stud Alcohol 1977;38:2025-35.

21 Benton A, Hamsher K, Varney N, Spreen O. Contributions to Neuropsychological Assessment: A Clinical Manual. New York: Oxford University Press, 1983.

22 Dixon WJ, Brown M, Engelman L, et al. BMDP statistical software. Los Angeles: University of California Press, 1983.

23 Ray A. SAS User's Guide: Basics 1982 edition. Cary NC: SAS Institutes, 1982.

24 Brown RG, Marsden CD, Quinn N, Wyke MA. Alterations in cognitive performance and affect-arousal state during fluctuations in motor function in Parkinson's disease. J Neurol Neurosurg Psychiatry 1984;47:454-65.

25 Riklan M, Whelihan W, Cullinan T. Levodopa and psychometric test performance in Parkinsonism-5 years later. Neurology 1976;26:173-9.

26 Delis D, Direnfeld L, Alexander MP, Kaplan E. Cognitive fluctuations associated with the on-off phenomenon in Parkinson disease. Neurology 1982;32:1049-52.

27 Girotti F, Carella F, Grassi MP, Soliveri P, Marano R, Caraceni T. Motor and cognitive performances of Parkinsonian patients in the on and off phases of the disease. $J$ Neurol Neurosurg Psychiatry 1986;49:657-60.

28 Weingartner $\mathrm{H}$. Psychobiological determinants of memory failure. In: Squire L, Butters N, eds. Neuropsychology of Memory. New York: Guilford Press, 1984:203-12.

29 Oades RD. Impairments of search behavior in rats after haloperidol treatment, hippocampal or neocortical damage suggest a mesocorticolimbic role in cognition. Biol Psychol 1981;12: 77-85.

30 Sahakian BJ, Sarna GS, Kantamaneni BD, Jackson A, Hutson PH, Curzon G. Association between learning and cortical catecholamines in non-drug treated rats. Psychopharmacology (Berl) 1985;86:339-43.

31 Javoy-Agid F, Agid Y. Is the mesocortical dopaminergic system involved in Parkinson disease? Neurology 1980;30:1326-30.

32 Price KS, Farley IJ, Hornykiewicz D. Neurochemistry of Parkinson's disease: Relation between striatal and limbic dopamine. Adv Biochem Psychopharmacol 1978;19:293-300. 